\title{
Neues in der Behandlung neurologischer Notfälle
}

\author{
M. Maschke; K. Schröder
}

Abteilung für Neurologie und Neurophysiologie des Krankenhauses der Barmherzigen Brüder Trier

\section{Schlüsselwörter}

Maligner Mediainfarkt, intrazerebrale Blutung, bakterielle Meningitis, virale Enzephalitis, Intensivneurologie

\section{Zusammenfassung}

Innovationen: Akute neuromuskuläre Erkrankungen: Rituximab führt bei einer therapierefraktären Myasthenie häufig zu einer signifikanten Verbesserung. Studien weisen auf eine mögliche Wirkung von Eculizumab hin. Bei der Behandlung des Guillain-Barré-Syndroms sind Plasmapherese und Immunglobuline gleichwertig, die Immunadsorption eine gute Alternative. Kortikosteroide scheinen die Prognose zu verschlechtern. Ischämische Schlaganfälle: Neuerungen sind positive Studien zur Thrombektomie. Die Destiny-II-Studie wies bei Patienten über dem 70. Lebensjahr mit einem malignen Mediainfarkt eine Senkung der Mortalität durch eine Hemikraniektomie nach. Intrazerebrale Blutungen: Die INTERACT-2-Studie wies keine sichere Überlegenheit einer aggressiven Blutdrucksättigung nach. In der Therapie der intrazerebralen Blutung ist der STICH-II-Trial zur operativen Therapie von Lobärhämatomen abgeschlossen, eine frühe Operation zeigte keinen eindeutigen Vorteil. Die SAIVMs-Studie wies einen Nachteil der operativen Exzision von Kavernomen gegenüber einem konservativen Vorgehen bezüglich des Outcomes nach 5 Jahren nach. Gleiches gilt für unrupturierte arteriovenöse Malformationen. Meningitis und Enzephalitis: Neuere Studien zeigen einen Rückgang der Inzidenz der Meningitis durch Meningokokken und Pneumokokken.

Korrespondenzadresse

Prof. Dr. Matthias Maschke

Abteilung für Neurologie und Neurophysiologie

Brüderkrankenhaus Trier

Nordallee 1, 54292 Trier

Tel. 0651/2082741, Fax 0651/2082749

m.maschke@bk-trier.de
Die Dexamethason-Gabe bei bakterieller Meningitis ist nach den aktualisierten DGN-Leitlinien nur noch nach einer nachgewiesenen Pneumokokkenmeningitis empfohlen. Bei anderen bakteriellen Meningitiden sollte jedoch nach mikrobiologischen Resultaten Dexamethason abgesetzt werden. Bezüglich viraler Enzephalitiden ist die zusätzliche Gabe von Valaciclovir nach einer intravenösen Gabe von Aciclovir bei Herpes-simplex-Enzephalitis nicht prognoseverbessernd. In Serbien wurden mehr als 50 Fälle mit einer West-Nil-Virus-Enzephalitis nachgewiesen, ebenso erste Fälle in Österreich, sodass das Virus in die Differenzialdiagnose einbezogen werden muss. Das in Polynesien, Südamerika und in der Karibik endemisch vorkommende Zikah-Virus führt häufig zu einem parainfektiösen Guillain-Barré-Syndrom, seltener zu einer Meningoenzephalitis.

\section{Keywords}

Malignant brain infarction, intracerebral hemorrhage, bacterial meningitis, viral encephalitis, neurological intensive care medicine

\section{Summary}

Innovations: Acute neuromuscular disease: Rituximab significantly improve treatment-refractory Myasthenia gravis. Studies hints at a possible efficacy of eculizumab. Plasmapheresis and i.v. Immunoglobulins appear to have the same efficacy in treatment of acute Guillain-Barré syndrome, immunadsorption is a good alternative. In contrast, corticosteroids impair prognosis in GBS. Ischaemic stroke: Sev-

Update of treatment in neurological emergencies Nervenheilkunde 2016; 35: 680-688

eingegangen am: 20. Mai 2016

angenommen am: 1. Juni 2016 eral positive randomized studies proved efficacy of thrombectomy. Thus, thrombectomy with stent retrievers after bridging thrombolysis is evidence based. The Destiny II trial revealed a significant decrease of mortality by hemicraniectomy in patients older than 70 years with malignant stroke in the medial cerebral artery territory. Intracerebral hemorrhage: INTERACT2 trial showed no significant improvement with aggressive treatment of arterial hypertension in comparison to conservative approaches to decrease arterial hypertension in patients with acute intracerebral hemorrhage. STICH-II trial investigated whether early surgical treatment is superior to conservative treatment in patients with lobar haematoma. The trial revealed no difference between both treatment approaches. SAIVMs trial showed a better outcome of conservative vs. surgical treatment in patients with intracranial cavernous after 5 years. A similar result was found for unruptured intracranial arteriovenous malformations. Meningitis and encephalitis: Studies showed a decrease in incidence of meningococcal and pneumococcal meningitis. Administration of dexamethasone in patients with acute bacterial meningitis is recommended only for patients with pneumococcal meningitis according to DGN guidelines. In acute meningitis due to other bacteria it should be withdrawn after microbiological results are received. In herpes simplex virus encephalitis the additional administration of valaciclovir after i.v. aciclovir has no influence on outcome. In Serbia 50 cases of West Nil virus encephalitis have been detected, other cases have been revealed in Austria hinting at inclusion of this virus in the differential diagnosis of encephalitis in Europe. Very recently, the Zikah virus which is endemic in Polynesia, South America and Caribbean regions has been shown to cause parainfectious Guillain-Barré syndrome, rarely meningoencephalitis. 
In den vergangenen drei Jahren sind interessante Studien zu unterschiedlichen notfallmäßig zu versorgenden neurologischen und neuropsychiatrischen Erkrankungen publiziert worden. Am wichtigsten für den klinischen Alltag sind die 2015 veröffentlichten positiven Studien (MR CLEAN, REVASCAT, ESCAPE, EXTEND 1A und SWIFT PRIME) zur Thrombektomie bei Schlaganfällen, die im starken Gegensatz $\mathrm{zu}$ den negativen Studien aus dem Jahr 2013 stehen. Zudem erregten die Studien zur Akutbehandlung von intrazerebralen Blutungen wie STICH-II und Interact-2 sowie die DESTINY-II-Studie zur Hemikraniektomie bei über 70-jährigen Patienten Aufmerksamkeit. Hauptaugenmerk soll bei der vorliegenden Zusammenfassung auf die häufigsten neurologischen Notfälle gelegt werden.

\section{Notallsituation}

\section{Akute Para- und Tetraparese Myasthenia gravis}

Patienten mit anti-Musk-positiver oder seronegativer generalisierter Myasthenia gravis scheinen insgesamt eine schlechtere Prognose und ein geringeres Ansprechen auf die Therapie zu haben als Patienten mit AChR-Ab-positiver Myasthenia gravis. Neuere Studien zeigten bei bis zu 50\% der vormals seronegativen Patienten mit Myasthenia gravis geclusterte Antikörper gegen Acetycholinrezeptoren, die sich vorwiegend bei generalisierter Erkrankung, aber auch bei okulärer Myasthenie nachweisen ließen (41). Eine weitere klinische Studie deckte eine überzufällig häufige Assoziation einer Myasthenia gravis mit Neuromyelitis optica (NMO) Spektrumerkrankungen auf (53). Dabei trat die Myasthenia gravis in 14 von 16 Patienten vor Beginn der Aquaporin-Antikörper positiven NMO auf.

Die myasthene Krise ist eher eine seltene Erkrankung geworden, hat aber insbesondere bei älteren Patienten immer noch eine hohe Mortalität. Ähnlich wie beim Gullain-Barré-Syndrom gibt es schon lange eine Diskussion, ob eine Immunglobulingabe (ivIg) oder eine Plasmapherese in der Akuttherapie besser und rascher anschlägt. Lange Zeit fehlten befriedigende randomi- sierte Studien. Barth et al. (6) veröffentlichten 2011 eine doppelblind-randomisierte Studie mit 84 Patienten mit einer myasthenen Krise (Quantitative Myasthenia Gravis Score $>10,5+$ zunehmende Schwäche). Die Hälfte erhielten Immunglobuline (1 $\mathrm{g} / \mathrm{kgKG}$ Gammunex über 2 Tage), die andere Hälfte wurde an 5 Tagen hintereinander plasmapheriert. Nach 14 Tagen gab es hinsichtlich des primären Endpunktes (Änderung des QMGS, 69\% der Patienten mit ivIg, 65\% der Patienten mit Plasmapherese verbesserten sich) keine Unterschiede. Auch die Verträglichkeit war nicht unterschiedlich. Das Vorhandensein von Acetylcholinrezeptorantikörpern und ein höherer QMGS bei Baseline waren positive Prädiktoren für ein Ansprechen auf die Therapie. Eine weitere Untersuchung unterstützte diese Ergebnisse und wies nach, dass die Kosten für die Therapie mit ivIg geringer sind als die der Plasmapherese (53.801 USDollar vs. 33.924 US-Dollar) (58).

Neuere Fallsammlungen unterstützen die aus älteren retrospektiven Analysen bekannte Wirksamkeit von Rituximab in der Behandlung der therapierefraktären Myasthenia gravis, insbesondere auch der AntiMuSK-Antikörper-positiven Myasthenia gravis (22). Das Rituximab führte dabei selten zu schwerwiegenden Nebenwirkungen und bei 7 von 13 behandelten therapierefraktären Patienten zu einem kompletten Ausschleichen des Kortisons.

Mycophenolatmofetil (CellCept $\left.{ }^{\circ}\right)$ wird, obwohl die Studien widersprüchlich sind, zur immunsuppressiven, kortisonsparenden Therapie der generalisierten Myasthenie empfohlen. Mittlerweile hat der Gemeinsame Bundesausschuss mit Beschluss vom 19.09.2013 eine Off-label-Indikation für Mycophenolatmofetil bei der Therapie der Myasthenia gravis als positiv beschieden, wobei eine Unverträglichkeit oder Unwirksamkeit von Azathioprin nachgewiesen sein muss. Eine andere, neue Alternativ stellt der monoklonale Antikörper Eculizumab dar, der in einer doppelblindrandomisierten Phase-II-Studie bei therapierefraktärer Myasthenia gravis positiv war (38).

Zudem gab es Hinweise, dass Patienten mit Myasthenia gravis häufig einen signifikanten Vitamin-D-Mangel aufweisen (2). Die Supplementation von Vitamin $\mathrm{D}_{3}$ führt zu einer Verbesserung der immunologischen Antwort auf die Therapie und zu einer verminderten myasthenieassoziierten Fatigue.

\section{Guillain-Barré-Syndrom und verwandte Erkrankungen}

Die Inzidenz des Guillain-Barré-Syndroms beläuft sich in der westlichen Welt zwischen 0,89 und 1,89 Fällen pro 100000 Personen pro Jahr. Ein Anstieg der Inzidenz um 20\% alle 10 Jahre nach der ersten Lebensdekade wird ebenfalls beobachtet. Möglicherweise führt die Impfung gegen H1N1 zu einem leicht erhöhten Risiko (geimpfte Personen: 1,92/100000 vs. 1,22/100000 bei ungeimpften Personen) (18). Letztendlich ist unter Zusammenschau aller erhältlichen Daten das Risiko deutlich höher durch eine typische Influenza oder H1N1-Infektion ein GBS zu bekommen als durch eine Impfung $(34,52)$. Interessanterweise scheinen die axonalen Varianten (AMAN) eher mit einer vorausgegangenen Durchfallerkrankung, wahrscheinlich durch Campylobacter jejuni, bedingt zu sein als durch eine Influenza oder andere virale Infektion (12).

Eine größere Fallsammlung zeigte bei Patienten mit GBS, die bereits intensivpflichtig waren, dass eine Immunadsorption sicher angewandt werden kann und dass die sequenzielle Therapie mit Immunadsorption und nachfolgenden Immunglobulinen keinen Vorteil gegenüber der alleinigen Immunadsorption $\mathrm{zu}$ haben scheint (30). Bei der Gabe der Immunglobuline muss beachtet werden, dass individuell eine sehr unterschiedliche Pharmakokinetik vorhanden ist und dass dies die Prognose beeinflusst. Eine holländische Studie zeigte, dass Patienten mit einem geringen Anstieg des Serum IgG von einer erhöhten Tagesdosis profitieren könnten (50). Des Weiteren scheint die nicht seltene Praxis bei wenig betroffenen, gehfähigen Patienten vor der Gabe von Immunglobulinen einen Therapieversuch mit Kortikosteroiden zu unternehmen zwecklos und eventuell sogar schädlich (40). Möglicherweise könnte die Verschlechterung der Prognose durch Kortikosteroide auch mit der Induktion erhöhter Blutzuckerwerte zusammenhängen. Darüber hinaus gibt es 
eine Diskussion, dass ein zu intensives körperliches Training zu einem schlechteren Outcome führen könnte. Eine kürzlich publizierte, randomisierte Studie widerlegte diese Studie und zeigte, dass Patienten nach einem GBS sehr wohl von einem möglichst intensiven rehabilitativen Programm profitieren (46).

Das dem GBS verwandte Miller-FisherSyndrom ist durch eine Ophthalmoplegie, schwere sensible Ataxie, Areflexie gekennzeichnet und weist in 83\% GQ1b-Antikörper und in 21\% Antikörper gegen Campylobacter jejuni auf. Die seltene BickerstaffEnzephalitis ist durch eine Bewusstseinsstörung, Areflexie und Ophthalmoplegie charakterisiert und weist GQ1b-Antikörper in 68\% und Antikörper gegen Campylobacter jejuni in 23\% auf. Dabei tritt nach einer epidemiologischen Studie die Bickerstaff-Enzephalitis etwa mit 100 Fällen/Jahr in ganz Japan auf, sodass die jährliche Inzidenz bei 1/1280000 Einwohner liegt (49). Lee et al. beschrieben eine neue Variante des MillerFisher-Syndroms, die Ophthalmoplegie ohne begleitende Ataxie (51). Dieses Syndrom hat ebenfalls in einem höheren Prozentsatz GQ1b-Antikörper nachweisbar. Im Vergleich zum Miller-Fisher-Syndrom sind eine isolierte vertikale Blickparese und eine interne Ophthalmoplegie häufiger. Die Entwicklung einer Bickerstaff-Enzephalitis scheint zudem durch eine aggressivere Störung der Bluthirnschranke (BHS) begünstigt zu sein, wobei eine solche Disruption der BHS bei der Miller-Fisher-Variante des GBS nicht gefunden wird (78).

\section{Elsberg-Syndrom und andere Polyradikulitiden}

Das Elsberg-Syndrom ist als Meningomyeloradikulitis der Cauda equina und des Konus beschrieben und als eigenständige Erkrankung umstritten. Benannt ist es nach dem New Yorker Neurochirurgen Charles Elsberg. An sich versteht man unter dem Elsberg-Syndrom eine entzündliche Erkrankung der sakralen Fasern mit starker Eiweißerhöhung im Liquor und einer Infektion mit HSV Typ 2 als häufige Ursache. Neuere Studien wiesen jedoch auch andere teilweise seltene Erreger wie Angiostrongylus cantonensis und humanes Herpesvirus Typ 2 (HHV 2) und eine Häufung bei HIV- positiven Patienten nach. Ähnlich der Mollaretschen Meningitis kann die Erkrankung bei HSV-Typ-2-Infektion rezidivieren, sodass eine prophylaktische Therapie notwendig wird (9). Mehrere Studien wiesen darauf hin, dass eine Therapie z. B. Valaciclovir auch zu einer Reduktion der HIV-Last im Serum führen kann. Momentan laufen Studien, die prospektiv den Einfluss der Therapie der HSV-Typ-Infektion auf die Progression der gleichzeitig vorliegenden HIV-Infektion untersuchen.

\section{Akuter Verwirrtheitszustand}

Nach ICD-Kriterien (F05) ist der akute Verwirrtheitszustand ein nicht durch Alkohol bedingtes mit gleichzeitig bestehender Störung von Bewusstsein, Aufmerksamkeit, Gedächtnis, Wahrnehmung, Denken, Psychomotorik, Emotionalität und einem geänderten Schlaf-WachRhythmus. Dabei wird ein akutes Auftreten, eine Zeitdauer unter 6 Monaten und deutliche Fluktuationen im Tages- und Wochenverlauf gefordert. Verwirrtheitszustände finden sich bei $14 \%$ bis $65 \%$ aller hospitalisierten Patenten über 80 Jahre, wobei medikamentöse Nebenwirkungen in 22\% bis 39\% aller Verwirrtheitszustände verantwortlich sind (4). Nach einer Studie von Hufschmidt et al. sind Parkinsonmedikamente, Diuretika, trizyklische Antidepressiva und Benzodiazepine für 18,5\% aller akuten Verwirrtheitszustände verantwortlich (39). Antihistaminika wie Ranitidin sind ebensfall nicht selten Ursache für delirante Zustände, sodass Protonenpumpenhemmer in der Vermeidung von gastralen Ulzera bevorzugt werden sollten (29). Daneben sind postoperative Verwirrtheitszustände besonders häufig und treten im Mittel bei 40\% (9\%-87\%) aller Patienten nach Interventionen auf (45). In der Pathophysiologie des Delirs spielen Änderungen der Neurotransmitter sicherlich eine sehr große Rolle. Studien weisen jedoch auf entzündliche Mechanismen wie eine Mikrogliaaktivierung und Zytokinausschüttung wie Interleukin-6 hin (65). Trotz zahlreicher Studien und Therapieempfehlungen scheint das Wissen noch nicht in ausreichendem Masse auf Intensivstationen angekommen zu sein, wie Untersuchungen suggerieren (69).

\section{Transiente globale Amnesie}

Die transiente globale Amnesie (TGA) ist durch eine vorübergehende isolierte Störung des retro- und anterograden Gedächtnisses für rezente, frische Informationen ohne weitere assoziierte Symptome gekennzeichnet. Typischerweise stellen die Betroffenen innerhalb weniger Minuten immer wieder die gleichen Fragen, wirken ratlos und restituieren komplett innerhalb von 24 Stunden. Bei Personen über dem 50. Lebensjahr beträgt die Inzidenz immerhin 20-30 Fälle/100000 Einwohner/Jahr. Rezidive sind selten, kommen aber bei bis zu 5\% der Patienten vor. Die Prognose im Hinblick auf die Merkfähigkeit im weiteren Verlauf nach der TGA ist dabei gut. Nachdem in früheren Jahren die Bildgebung immer als normal beschrieben worden war, zeigten neuere Untersuchungen, dass es häufig zu einer hippocampalen Diffusionsstörung im CA1-Feld des Ammonshorns kommt (7). Wichtig in der Bildgebung scheint die Schichtdicke zu sein, da die Sensitivität mit Zunahme der Schichtdicke abnimmt (44,7\% für $3 \mathrm{~mm}, 27,1 \%$ für $5 \mathrm{~mm}$ und 19,6\% für $6 \mathrm{~mm}$ ). Dabei scheint ein metabolischer Stress der CA1-Neurone eine wichtige Rolle in der Pathophysiologie zu spielen (8). Die Assoziation der TGA mit Pathologien der zerebralen Venen oder Jugularvenen scheint eher zufällig, sodass nach wie vor unklar bleibt, wodurch die TGA verursacht wird (5). Auffällig ist eine Assoziation mit akuten internistischen Erkrankungen, z. B. Myokardinfarkten oder anderen akuten Herzerkrankungen $(11,23)$.

\section{Akute Bewusstseinsstörung}

\section{Status epilepticus}

Der Status epileticus hat eine signifikante Mortalität. Insbesondere beim therapierefraktären Status steigt die Mortalität bis auf $39 \%$ an. Bei pädiatrischen Patienten scheint die Gabe von Lorezapam $0,1 \mathrm{mg} / \mathrm{kg}$ i.v. vs. Diazepam $0,2 \mathrm{mg} / \mathrm{kg}$ in Effektivität und Nebenwirkungen vergleichbar zu sein (19). Zudem wurde in einer kleineren Studien beim therapierefraktären Status die Wirksamkeit von Propofol gegen Barbiturate getestet (77). Die Wirksamkeit und Nebenwirkungen erschienen gleich, die Beatmungsdauer war in der Barbiturat- 
gruppe jedoch signifikant länger. Problem für den Einsatz von Propofol ist jedoch das Propofol-Infusionssyndrom, welches in einer anderen retrospektiven Analyse bei einem von 18 Patienten auftrat (74). Levetiracetam scheint nach randomisierten Studien ebenfalls eine ähnliche Wirksamkeit wie Lorazepam aufzuweisen und führt möglicherweise zu einer geringeren Hypotension und geringerer Intubationsrate (62). Bei fokalem Status epilepticus oder Status nonconvulsivus kann der Einsatz von intravenösem Lacosamid erwogen werden. Die Ansprechrate wird allerdings in retrospektiven Analysen mit 68\% angegeben (80).

\section{Basilaristhrombose}

Standardvorgehen in der Therapie der Basilaristhrombose war über Jahre die intraarterielle Lyse mit rtPA. Problem war häufig, dass die Therapie zu spät begonnen wurde aufgrund einer zu langen Prähospitalphase, manchmal jedoch auch wegen einer zu langen Door-to-needle-Zeit, bedingt durch infrastrukturelle Probleme. Patienten mit einer Basilaristhrombose, die innerhalb von 6 Stunden i.a. lysiert werden, weisen immerhin in $60 \%$ ein gutes Outcome mit einem mRS von 0-2 auf, Patienten mit Lysebeginn nach 6 Stunden nur noch in etwa $20 \%$. Insgesamt haben noch $68 \%$ der Patienten ein schlechtes Outcome. Problem ist, dass randomisierte Studien fehlen, die die neuen interventionellen Techniken gegenüber einer i.v.-Lyse gerade in Bezug auf die Basilaristhrombose untersuchen. Gleiches gilt für BridgingKonzepte, bei der zunächst eine i.v.-Lyse begonnen wird und bei Fehlschlagen dieser im zweiten Schritt interventionell vorgegangen wird. Kleine Fallsammlungen weisen darauf hin, dass dieses BridgingKonzept durchaus erfolgreich sein könnte (71). Ein weiteres Problem ist die Vorhersagekraft klinischer Zeichen für das Outcome nach Lyse. Bisher war ein komatöser Bewusstseinszustand zumindest ein relatives Ausschlusskriterium für die Lyse bei einer Basilaristhrombose. Eine kürzlich veröffentlichte Studie zeigte jedoch, dass die Glasgow Coma Scale (GCS) keine sehr gute Prädiktion für das Outcome nach interventioneller Behandlung einer Basila- risthrombose aufweist (20). Insbesondere bestand keine Korrelation zwischen dem GCS bei Aufnahme und dem modified Rankin Scale nach 90 Tagen.

\section{Meningitis und Enzephalitis}

\section{Akute bakterielle Meningitis}

Die wichtigste Studie zum Thema bakterielle Meningitis wurde 2002 veröffentlicht. Die Ergebnisse der Studie zeigten an einer größeren Patientenpopulation ( $\mathrm{n}=301)$, dass die Gabe von Dexamethason (4 x 10 mg iv. für 4 Tage, erste Gabe vor oder zeitgleich zur ersten Gabe des Antibiotikums) bei Patienten mit einer Pneumokokkenmeningitis signifikant die Mortalität reduziert und mit einem besseren funktionellen Outcome verbunden ist. Eine ergänzende Studie wurde 2007 veröffentlicht (67). Dabei erhielten wiederum alle Patienten $(n=435)$ mit dem Verdacht einer bakteriellen Meningitis Dexamethason oder Placebo. Insgesamt führte Dexamethason entgegen den Ergebnissen von de Gans et al. nicht zu einer Verbesserung des Outcomes. In der anschließenden Subgruppenanalyse zeigte sich, dass nur die Patienten von Dexamethason profitieren, die eine aus der Kultur bestätigte bakterielle Meningitis aufwiesen. Eine Studie zur Wirksamkeit von Dexamethason bei Patienten mit Meningokokkenmeningitis wies einen positiven Effekt auf die assoziierte Arthritis nach und zeigte keine negativen Effekt auf Taubheit, Glasgow Coma Scale oder Tod (36). Neuere Metaanalysen lassen erneut Zweifel an der Wirksamkeit der Gabe von Dexamethason in der Therapie der bakteriellen Meningitis aufkommen (14). Die aktualisierte Leitlinie der DGN sieht aber die direkte, zusätzliche Gabe von Dexamethason vor, da zumindest der Effekt bei Pneumokokkenmeningitis wahrscheinlich ist (72). Nach Eintreffen eines mikrobiologischen Ergebnisses, das eine Pneumokokkenmeningitis ausschließt, sollte Dexamethason jedoch abgesetzt werden. Zudem soll die empirische Antibiotikatherapie weiterhin bei zuvor gesunden Erwachsenen mit einem Cephalosporin der dritten Generation (in der Regel Ceftriaxon) und Ampicillin durchgeführt werden.

Eine weitere Frage, die nicht gut in Studien untersucht ist, ist die Dauer der Anti- biotikatherapie bei einer nachgewiesenen bakteriellen Meningitis. Eine Studie von Molyneux et al. zeigte, dass bei Kindern älter als die neonatale Periode eine Antibiotikatherapie mit Ceftriaxon bereits nach 5 Tagen beendet werden kann, wenn die Patienten bis dahin stabil sind. Bei Erwachsenen fehlen solche Studien, sodass die Dauer zumeist bei mindestens 10 Tagen (außer bei der länger zu behandelnden ListerienMeningitis) liegen wird (64).

\section{Virale Enzephalitis und Differenzialdiagnosen}

An der Therapie der Herpes-Enzephalitis hat sich nichts Wesentliches geändert. Neue Medikamente sind nur in der Behandlung von Enterovirus-Infektionen und Influenza dazugekommen. Darüber hinaus sind durch die ausgedehnte Reisetätigkeit der deutschen Bevölkerung „emerging viruses" wie West-Nil- oder Japan-Enzephalitis-Virus zu beachten. Nach den Ergebnissen der EuroTravNet 2010 (31) stieg unter den viralen Erkrankungen das Dengue-Fieber von 127 Fällen im Jahr 2008 innerhalb der EU auf 299 Fälle im Jahr 2010 an. Eine italienische Studie zeigte, dass das Vorkommen des West-Nil-Virus in Italien ansteigt und bei einer Inzidenz von 0,55 Fälle/100000 Einwohner auf 3 Jahre lag (76). Dabei verstarben 16\% der erkrankten Patienten. In Bezug auf die weltweit am häufigsten zu Todesfällen führende virale Enzephalitis, die JapanEnzephalitis, ist es durch die Impfung zu einer verbesserten Situation gekommen (28). Etwas beunruhigender ist allerdings, dass in Italien Viruspuren des Japan-Enzephalitis-Virus in Moskitos isoliert werden konnten (75).

Infektionen mit dem West-Nil-Virus (WNV) bleiben in den USA und Kanada immer noch eine der häufigsten Ursachen schwerer viraler Enzephalitiden. Zwischen 1999 und 2012 traten 16196 Fälle mit neuroinvasiven WNV-Infektionen auf, davon endeten 1549 tödlich (70). Mittlerweile sind in Kanada 979 ZNS-Infektionen durch WNV bestätigt. Der Virus wird dabei zumeist durch Moskitos übertragen, kann aber parenteral durch Blutprodukte oder Transplantate weitergegeben werden. Die Infektion kann sich in einer blanden Me- 
ningitis, einer Enzephalitis aber auch in einer schlaffen, Poliomyelitis-ähnlichen Tetraparese mit hoher Letalität äußern. Die Diagnose wird durch Nachweis von WNVIgM-Antikörpern in Liquor und/oder Serum bestätigt. Eine aktive Therapie gibt es ebenso wenig wie eine aktive Immunisierung. Schon länger war bekannt, das WNV bei Vögeln oder Pferden in Europa nachgewiesen werden kann. Beunruhigend sind jedoch neuere Untersuchungen, die autochthone Infektionen mit WNV bei Patienten mit Enzephalitis in Serbien (73) nachweisen konnten. Insofern muss damit gerechnet werden, dass es demnächst auch in Deutschland Patienten geben wird, die sich in Europa die Infektion mit WNV zugezogen haben.

2011 verstarben in relativ kurzem Zeitabstand in Sachsen-Anhalt drei Männer zwischen 62 und 63 Jahren an einer Meningoenzephalitis, die durch einen neuen Bornavirus ausgelöst wurde (37). Alle drei Patienten waren Züchter von Bunthörnchen, einer südamerikanischen Eichhörnchenart. Die Patienten wiesen klinisch Fieber, Schüttelfrost, eine zunehmende psychomotorische Verlangsamung, Verwirrtheit, zu Beginn ein unsicheres Gangbild, später Myoklonien, Störungen der Okulomotorik und eine zunehmende Bewusstseinsminderung bis hin zum Koma auf. Die zerebrale Kernspintomografie zeigte in der T2WI und FLAIR hyperintense Veränderungen in den Basalganglien und temporoparietalen und insulärem Kortex als auch des Hirnstamms, während das Rückenmark ausgespart blieb. 2 bis 4 Monate nach Beginn der Symptomatik verstarben die Patienten. In den anschließenden ausführlichen Post-mortem-Untersuchungen des Gewebes und der Körperflüssigkeiten inklusive Metagenomics, whole-genome sequencing, immunhistochemischer Untersuchungen und PCR-Techniken in der Neuropathologie in Magdeburg, am Friedrich-Löffler-Institut in Greifswald als auch am Bernhard-Nocht-Institut für Tropenmedizin wurde ein nicht beschriebenes, von Bunthörnchen übertragenes Bornavirus als Verursacher identifiziert (variegated squirrel-derived Bornavirus-1, VSBV-1). In einem der Patienten konnten Bornavirusspezifische IgG-Antikörper im Liquor und Serum nachgewiesen werden.
Der erste Fallbericht, der im Zusammenhang mit einer Zika-Virus-Infektion von einem akuten Gullain-Barré-Syndrom berichtete, stammt aus dem Jahr 2014 (68). Die dabei betroffene 40-jährige Polynesierin entwickelte nach einer kurzen Prodromalphase mit Parästhesien eine schlaffe Tetraparese und eine Dysautonomie. Die Elektrophysiologie zeigte typische Charakteristika mit F-Wellen-Verlängerungen und verlängerten distal motorischen Latenzen. Im Liquor fand sich ebenfalls ein typischer Befund mit einer zytoalbuminären Dissoziation. Im Labor waren lediglich die Transaminasen leicht erhöht. Unter einer Immunglobulintherapie über 5 Tage kam es zu einer raschen Besserung, sodass die Patientin nach 13 Tagen entlassen werden konnte. Etwa 7 Tage vor Beginn der neurologischen Symptomatik litt die Patientin an grippeähnlichen Symptomen mit Myalgien, Fieber und einer Konjunktivitis. Am Tag 1 der neurologischen Symptome war die RT-PCR auf Zika-Viren noch negativ, am Tag 8 und 28 zeigten sich dann die Zika-spezifischen IgM- und IgG-Antikörper jedoch positiv. Insgesamt konnte in Polynesien seit der Zika-Virus-Epidemie mit 8200 nachgewiesenen Zika-Virus-Fällen in Polynesien eine Zunahme der Inzidenz des GBS um das 20-fache beobachtet werden, sodass ein Zusammenhang zwischen dem Virus und Polyneuritiden sehr nahe liegt (68).

Zanamivir und Oseltamivir sind für die Behandlung der Influenza zugelassen. Zanamivir $\left(\right.$ Relenza $\left.^{\circ}\right)$ ist ein Inhibitor der Neuroaminidase. Die Neuroaminidase wird vom Virus benötigt, um von infizierten Zellen zu gesunden Zellen zu wechseln. Zanamivir hemmt die Ausbreitung von Influenza-Virus-Typ-A und -B im Körper. Dadurch vermindert es die Symptome und die Dauer der Erkrankung. Die Behandlung sollte so früh wie möglich, innerhalb von 48 Stunden nach Einsetzen der Symptome, beginnen. Die empfohlene Dosis beträgt 2-mal täglich 2 Inhalationen über 5 Tage. Dies entspricht einer Gesamttagesdosis von $20 \mathrm{mg}$. Oseltamivir (Tamiflu $\left.{ }^{\circledR}\right)$ ist ebenfalls ein Neuroaminidaseinhibitor. Der wichtigste Unterschied zum Zanamivir, das inhaliert wird, ist die perorale Applikationsweise von Oseltamivir. Nach einer Studie scheint Zanamivir in der Behandlung der Influenza-TypB-Infektion dem Oseltamivir überlegen zu sein (44). Einzelfallberichte weisen jedoch darauf hin, dass Oseltamivir eine Wirksamkeit in der Behandlung der durch Influenza-Typ-B-Virus bedingten Enzephalitis haben könnte (79).

Plecoranil (Picovir, WIN 63 843) ist ein noch nicht zugelassenes Virustatikum mit Wirksamkeit gegen Enteroviren und Rhinoviren. Es ist ein oral applizierbarer Kapsidblocker, der mit dem Kapsid von Rhinoviren und Enteroviren interagiert. Die Kapsidstruktur verändert sich durch Plecoranil und es kommt zum Uncoating und zur Freisetzung der viralen RNA in das Zytoplasma, wo die Replikation der Viren erfolgt. Eine neuere Studie lässt einen milden Effekt mit einer Verkürzung der Erkrankungsdauer bei Kindern mit Enterovirus Meningitis erkennen (25).

Ansonsten sehen die revidierten Leitlinien zur Therapie viraler Meningoenzephalitiden weiterhin vor, dass bei enzephalitischer Symptomatik und dem Verdacht auf eine Herpesvirus-Ätiologie die i.v.-Gabe von Aciclovir ohne Verzug einzuleiten ist. Eine Wirksamkeit von Kortikoiden als begleitende Therapie der Herpes-Enzephalitis ist nicht erwiesen und Gegenstand laufender Studien (61), auch wenn einzelne Fallberichte einen positiven Effekt nahelegen (54). Zudem wies eine dieses Jahr veröffentlichte Studie nach, dass die zusätzliche Gabe von Valaciclovir im Anschluss an die Akuttherapie mit Aciclovir keine Wirksamkeit hat (33).

Eine weitere rezente Studie zeigte, dass VZV-Antigen bei 74\% der RZA-positiven Temporalarterien von Patienten mit Verdacht auf eine Arteriitis temporalis nachgewiesen werden kann (32). Bei normalen Temporalarterien war VZV-Antigen nur in $8 \%$ positiv. Diese Studie legte eindeutig nahe, dass VZV-Infektionen eine Rolle in der Pathogenese der Arteriitis temporalis spielen. Dabei fand sich VZVAntigen in der Nähe oder direkt in den entzündeten Stellen in allen Schichten der Arterie mit Bevorzugung der Adventitia. Dies impliziert eine transaxonale Ausbreitung ausgehend von den Ganglien der Hirnnerven ähnlich wie bei VZV-Vaskulopathien. 


\section{Akuter Kopfschmerz}

\section{Akute intrazerebrale Blutung}

Im klinischen Alltag ist es manchmal schwierig, die Prognose bei Patienten mit einer intrazerebralen Blutung (ICB) vorherzusagen. Die PREDICT-Studie untersuchte an 268 Patienten das "spot sign“, welches in der CT-Angiografie bei einem Teil der Patienten mit einer ICB sichtbar wird (24). Die mediane Hämatom-Zunahme betrug für die „spot-sign“ positiven Patienten $8,6 \mathrm{ml}$ vom ersten CT zum Followup-CT, während die „spot-sign“ negativen Patienten lediglich eine Zunahme von 0,4 $\mathrm{ml}$ aufwiesen. Somit zeigt das "spot-sign“ eine schlechtere Prognose an und könnte als Auswahlkriterium für zukünftige Interventionsstudien dienen. Eine weitere Studie deckt sich mit der alltäglichen Beobachtung, dass kleine intrazerebrale Hämatome seltener eine Hämatom-Expansion aufweisen als größere, wobei der Cut-offWert für eine schlechtere Prognose bei 10 ml Hämatomvolumen lag (27).

Mit der wichtigen Frage, ob eine frühe neurochirurgische Hämatomausräumung zu einer verbesserten Prognose bei Patienten mit intrazerebralen Blutungen führt, beschäftigte sich der multizentrische International Surgical Trial in Intracerebral Hemorrhage (STICH) (59). In dieser Studie wurden 1033 Patienten entweder frühzeitig (innerhalb der ersten 24 Stunden nach Randomisierung) neurochirurgisch ( $\mathrm{n}=$ 503) oder initial rein konservativ $(n=530)$ behandelt. Das entscheidende Ergebnis war, dass die Patienten mit einem guten Outcome gemessen an der Glasgow Outcome Scale nach 6 Monaten identisch zwischen beiden Gruppen war (26\% vs. $24 \%$, Odds Ratio OR 0,89). Dies belegt erstmals in einer randomisierten Studie mit ausreichender Patientenzahl, dass zunächst ein konservatives Vorgehen bei Patienten mit intrazerebralen Blutungen gewählt werden sollte. Nur bei jüngeren Patienten mit zunehmender Mittelllinienverlagerung oder oberflächennahen Lobärhämatomen kann zur Verbesserung der Prognose quo ad vitam ein primär operatives Vorgehen sinnvoll sein, wobei dies jedoch nichts am funktionellen Outcome ändert. Interessanterweise ist nach der Veröffentlichung des STICH-I-Trials zumindest in Großbritan- nien eine Tendenz zu weniger neurochirurgischen Operationen nach intrazerebraler Blutung zu beobachten (48). Die anschließende STICH-II-Studie ist mittlerweile abgeschlossen, wobei die Studie den Einfluss der frühen Operation bei oberflächlich gelegenen Lobärhämatomen untersuchte (60). Entgegen der aus der STICH I generierten Ansicht, dass Lobärhämatome eventuell von einer Frühoperation profitieren könnte, zeigte diese Studie, dass auch bei Lobärhämatomen die frühe Operation nur einen geringen Vorteil bietet (schlechtes Outcome konservatives Vorgehen 62\%, operatives Vorgehen 59\%, ARR 3,7\%, OR $0,86)$.

Die andere wichtige Studie, die zum Thema ICB veröffentlicht wurde, war die INTERACT-2-Studie (3). Die Studie untersuchte an 2839 Patienten mit akuter ICB, ob ein rasches und intensives Absenken der Blutdruckwerte zu einem besseren Outcome führt (Zielwert Gruppe 1: $<140 \mathrm{mmHg}$ am Tag 1, Zielwert Gruppe 2: $<180$ $\mathrm{mmHg}$ ). Obwohl die primäre Analyse keinen Benefit der intensiven Therapie auf Mortalität und Behinderung zeigte, wies eine Post-hoc-Analyse des Modified Rankin Scale auf einen möglichen positiven Einfluss hin. Letztendlich ändert diese Studie jedoch nicht etwas am praktischen Vorgehen entsprechend der Leitlinien. Eine weitere Studie, die ICH-Adapt-Studie, konnte nachweisen, dass eine rasche Blutdrucksenkung nicht zu einem verminderten zerebralen Blutfluss um das Hämatom herum führt und so keine Ischämischie provoziert (15).

Mehrere Studien untersuchten zudem das Vorgehen bei unrupturierten arteriovenösen Malformationen und bei Kavernomen. Beide Erkrankungen profitieren zunächst von einem konservativen Vorgehen, wobei ein besseres Outcome gegenüber der frühen Operation bis über 12 Jahre nachverfolgt werden kann $(1,63)$.

\section{Subarachnoidalblutung}

Bezüglich Magnesium kursierten lange Zeit kleinere Studien, die eine Wirksamkeit der intravenösen Applikation zur Vermeidung von Vasopasmen nahelegten. Eine holländische Studie mit 606 Patienten zeigte, dass intravenöses Magnesium das Out- come nach einer SAB nicht verbessert (26). 2008 wurde die Phase-II-Studie (CONSCIOUS-1) zu Clazosentan, einem Endothelinrezeptorantagonist, veröffentlicht. Die Ergebnisse waren dabei positiv und zeigten bei tolerablen Nebenwirkungen eine Reduktion von moderaten und schweren Vasospasmen, jedoch nur im Trend eine verminderte Mortalität und Morbidität (55). Die Phase-III-Studie (CONSCIOUS-2) wurde dieses Jahr veröffentlicht und zeigte keinen signifikanten Unterschied zwischen Verum und Placebo (56). Eine weitere Phase-III-Studie (CONSCIOUS-3) untersuchte, ob Clazosentan einen günstigen Einfluss auf das Outcome nach SAB in der Subgruppe der Patienten mit einem gecoilten Aneurysma hat (57). Die vorzeitig gestoppte Studie wies zwar eine geringere Rate an Vasopasmen unter Clazosentan nach, konnte aber keinen Unterschied im Outcome erkennen. Eine weitere randomisierte Phase-II-Studie untersuchte die Wirksamkeit eines höheren Hämoglobin $(\mathrm{Hb}>11,5 \mathrm{~g} / \mathrm{dl})$ bei Patienten mit einer SAB (66). Dabei wurden Patienten mit geringeren $\mathrm{Hb}$-Werten Erythrozyten-Konzentrate infundiert $(n=20)$ und diese Gruppe mit einer normalbehandelten Gruppe ( $n=22)$ verglichen. Nebenwirkungen wurden nicht signifikant häufiger in der Therapiegruppe registriert, die Outcome-Parameter waren statistisch nicht signifikant unterschiedlich, es gab aber einen Trend zugunsten der Therapiegruppe. Eine Phase-III-Studie ist nach Ansicht der Autoren sinnvoll. Kleinere Phase-I- und PhaseII-Studien wurden zudem zu Landiolol, einem Betablocker, und Tiopronin, einem neuroprotektiv wirkenden Medikament mit Verminderung der Konzentration von 3-Aminopropanal (neurotoxisch) durchgeführt. Die Ergebnisse sind dabei positiv, jedoch fehlen adäquat gepowerte Therapiestudien.

\section{Akute Hemiparese}

\section{Schlaganfall}

Beim Schlaganfall gab es sehr interessante Studien, die in den letzten 12 Monaten veröffentlicht wurden. Für große Aufregung sorgten die fünf Thrombektomie Studien (MR CLEAN, ESCAPE, EXTEND 1A, REVASCAT und SWIFT PRIME $(10,16,35$, 
42) die 2015 publiziert wurden und im starken Gegensatz zu den negativen Studien aus dem Jahr 2013 stehen (SYNTHESIS, IMS-III und MR Rescue (13, 21, 47).

Die SYNTHESIS-Studie, IMS-III-Studie und MR-RESCUE-Studie hatten den Nachteil, dass sie teilweise sehr lange Rekrutierungszeiten hatten und entweder gar keine Stent-Retriever oder nur sehr selten moderne Thrombektomiedevices benutzten. Zudem gab es in den Subgruppenanalysen doch durchaus Hinweise, dass schwer betroffene Patienten profitieren könnten. In den 2015 veröffentlichten Studien konnte eindeutig belegt werden, dass Patienten mit proximalen intrakraniellen Verschlüssen innerhalb eines 6 Stundenzeitfensters von der Thrombektomie profitieren. Die meisten in die Studien eingeschlossenen Patienten erhielten rtPA vor der Thrombektomie, sodass ein Bridging-Konzept in der Versorgung von Patienten mit akuten, großen Schlaganfällen Sinn macht. Eine gute Zusammenfassung der Studiendaten und der daraus abzuleitenden Maßnahmen findet sich in der Publikation von Campbell et al. (17).

Die weitere für Aufmerksamkeit sorgende Studie war die DESTINY-II-Studie, die die Wirksamkeit der Hemikraniektomie bei älteren Patienten über 70 Jahre nach einem malignen Mediainfarkt untersuchte (43). Dabei zeigte sich zwar eine signifikant reduzierte Mortalität, jedoch kein Vorteil gegenüber dem klinischen Outcome der überlebenden Patienten mit einem mRS von zumeist 4 oder 5 .

\section{Interessenkonflikt}

Prof. Matthias Maschke erhielt Honorare für Vorträge und Beratungstätigkeiten sowie Unterstützung von Fortbildungsveranstaltungen von Boehringer Ingelheim, Biogen Idec, Eisai, Genzyme, Ipsen Pharma, Merck Serono, Novartis, Pharm Allergan, Pfizer, Roche Pharma, Shire Deutschland, UCB Pharma GmbH. Dr. Kerstin Schröder hat keine Interessenkonflikte in dem $\mathrm{Zu}$ sammenhang mit den im Artikel dargestellten Inhalten.

\section{Literatur}

1. Al-Shahi Salman R, White PM, Counsell CE, du Plessis J, van Beijnum J, Josephson CB, Wilkinson T, Wedderburn CJ, Chandy Z, St George EJ, Sellar RJ, Warlow CP. Outcome after conservative management or intervention for unruptured brain arteriovenous malformations. JAMA 2014; 311: 1661-1669.

2. Askmark H, Haggård L, Nygren I, Punga AR. Vitamin D deficiency in patients with myasthenia gravis and improvement of fatigue after supplementation of vitamin D3: a pilot study. Eur J Neurol 2012; 19: 1554-1560.

3. Anderson CS, Heeley E, Huang Y, Wang J, Stapf C, Delcourt C, Lindley R, Robinson T, Lavados P, Neal B, Hata J, Arima H, Parsons M, Li Y, Wang J, Heritier S, Li Q, Woodward M, Simes RJ, Davis SM, Chalmers J. Rapid blood-pressure lowering in patients with acute intracerebral hemorrhage. N Engl J Med 2013; 368: 2355-2365.

4. Back C, Wittmann M, Haen E. Medikamentös induziertes Delir. Ther Umsch 2011; 68: 27-33.

5. Baracchini C, Tonello S, Farina F, Viaro F, Atzori $\mathrm{M}$, Ballotta E, Manara R. Jugular veins in transient global amnesia: innocent bystanders. Stroke 2012; 43: 2289-2292.

6. Barth D, Nabavi Nouri M, Ng E, Nwe P, Bril V. Comparison of IVIg and PLEX in patients with myasthenia gravis. Neurology 2011; 76: 2017-2023.

7. Bartsch T, Deuschl G. Transient global amnesia: functional anatomy and clinical implications. Lancet Neurol 2010; 9: 205-214.

8. Bartsch T, Alfke K, Wolff S, Rohr A, Jansen O, Deuschl G. Focal MR spectroscopy of hippocampal CA-1 lesions in transient global amnesia. Neurology 2008; 70: 1030-1035.

9. Berger JR, Houff S. Neurological complications of herpes simplex virus type 2 infection. Arch Neurol 2008; 65: 596-600.

10. Berkhemer OA, Fransen PS, Beumer D et al. A randomized trial of intraarterial treatment for acute ischemic stroke. N Engl J Med 2015; 372: 11-20.

11. Bobinger T, Köhrmann M, Raaz-Schrauder D, Schwab S, Kallmünzer B. Lost memories can break your heart: a case report of transient global amnesia followed by takotsubo cardiomyopathy. Clin Res Cardiol 2013; 102: 693-696.

12. Brain S, Webb A, Zentar M, Turner MR. Seasonal variation, sub-type and prodromal illnesses in guillain-barre syndrome. J Neurol Neurosurg Psychiatry 2013; 84: e2.

13. Broderick JP, Palesch YY, Demchuk AM, Yeatts SD, Khatri P, Hill MD, Jauch EC, Jovin TG, Yan B, Silver FL, von Kummer R, Molina CA, Demaerschalk BM, Budzik R, Clark WM, Zaidat OO, Malisch TW, Goyal M, Schonewille WJ, Mazighi M, Engelter ST, Anderson C, Spilker J, Carrozzella J, Ryckborst KJ, Janis LS, Martin RH, Foster LD, Tomsick TA. Endovascular therapy after intravenous t-PA versus t-PA alone for stroke. $\mathrm{N}$ Engl J Med 2013; 368: 893-903.

14. Brouwer MC, McIntyre P, de Gans J, Prasad K, van de Beek D. Corticosteroids for acute bacterial meningitis. Cochrane Database Syst Rev 2010; 9: CD004405.
15. Butcher KS, Jeerakathil T, Hill M, Demchuk AM, Dowlatshahi D, Coutts SB, Gould B, McCourt R, Asdaghi N, Findlay JM, Emery D, Shuaib A. The Intracerebral Hemorrhage Acutely Decreasing Arterial Pressure Trial. Stroke 2013; 44: 620-626.

16. Campbell BC, Mitchell PJ, Kleinig et al. Endovascular therapy for ischemic stroke with perfusionimaging selection. N Engl J Med. 2015; 372: 1009-1118.

17. Campbell BC, Donnan GA, Lees KR, Hacke W, Khatri P, Hill MD, Goyal M, Mitchell PJ, Saver JL, Diener HC, Davis SM. Endovascular stent thrombectomy: the new standard of care for large vessel ischaemic stroke. Lancet Neurol 2015; 14: 846-854.

18. Centers for Disease Control and Prevention (CDC). Preliminary results: surveillance for Guillain-Barré syndrome after receipt of influenza $\mathrm{A}$ (H1N1) 2009 monovalent vaccine - United States, 2009-2010. MMWR Morb Mortal Wkly Rep 2010; 59: 657-661.

19. Chamberlain JM, Okada P, Holsti M, Mahajan P, Brown KM, Vance C, Gonzalez V, Lichenstein R, Stanley R, Brousseau DC, Grubenhoff J, Zemek R, Johnson DW, Clemons TE, Baren J. Lorazepam vs diazepam for pediatric status epilepticus: a randomized clinical trial. JAMA 2014; 311: 1652-1660.

20. Chandra RV, Law CP, Yan B, Dowling RJ, Mitchell PJ. Glasgow coma scale does not predict outcome post-intra-arterial treatment for basilar artery thrombosis. AJNR Am J Neuroradiol 2011; 32: 576-580.

21. Ciccone A, Valvassori L, Nichelatti M, Sgoifo A, Ponzio M, Sterzi R, Boccardi E. Endovascular treatment for acute ischemic stroke. $\mathrm{N}$ Engl J Med 2013; 368: 904-913.

22. Collongues N, Casez O, Lacour A, Tranchant C, Vermersch P, de Seze J, Lebrun C. Rituximab in refractory and non-refractory myasthenia: A retrospective multicenter study. Muscle Nerve 2012; 46: 687-691.

23. Courand PY, Sibellas F, Gonidec S, Mechtouff L, Kirkorian G, Bonnefoy E. Acute myocardial infarction: a precipitating event for transient global amnesia. J Cardiovasc Med (Hagerstown) 2014; 15: 78-79.

24. Demchuk AM, Dowlatshahi D, Rodriguez-Luna D, Molina CA, Blas YS, Dzialowski I, Kobayashi A, Boulanger JM, Lum C, Gubitz G, Padma V, Roy J, Kase CS, Kosior J, Bhatia R, Tymchuk S, Subramaniam S, Gladstone DJ, Hill MD. Prediction of haematoma growth and outcome in patients with intracerebral haemorrhage using the CT-angiography spot sign (PREDICT): a prospective observational study. Lancet Neurol 2012; 11: 307-314.

25. Desmond RA, Accortt NA, Talley L, Villano SA, Soong SJ, Whitley RJ. Enteroviral meningitis: natural history and outcome of pleconaril therapy. Antimicrob Agents Chemother 2006; 50: 2409-2414.

26. Dorhout Mees SM, Algra A, Vandertop WP, van Kooten F, Kuijsten HA, Boiten J, van Oostenbrugge RJ, Al-Shahi Salman R, Lavados PM, Rinkel GJ, van den Bergh WM. Magnesium for aneurysmal subarachnoid haemorrhage (MASH-2): a randomised placebo-controlled trial. Lancet 2012; 380: 44-49. 
27. Dowlatshahi D, Smith EE, Flaherty ML, Ali M, Lyden P, Demchuk AM. Small intracerebral haemorrhages are associated with less haematoma expansion and better outcomes. Int J Stroke 2011; 6: 201-206.

28. Fischer M, Lindsey N, Staples JE, Hills S. Japanese encephalitis vaccines: recommendations of the Advisory Committee on Immunization Practices (ACIP). MMWR Recomm Rep 2010; 59(RR-1): $1-27$.

29. Fujii S, Tanimukai H, Kashiwagi Y. Comparison and analysis of delirium induced by histamine $\mathrm{h}(2)$ receptor antagonists and proton pump inhibitors in cancer patients. Case Rep Oncol 2012; 5: 409-412.

30. Galldiks N, Dohmen C, Neveling M, Fink GR, Haupt WF. Selective immune adsorption treatment of severe Guillain Barré syndrome in the intensive care unit. Neurocrit Care 2009; 11: 317-321.

31. Gautret P, Cramer JP, Field V, Caumes E, Jensenius M, Gkrania-Klotsas E, de Vries PJ, Grobusch MP, Lopez-Velez R, Castelli F, Schlagenhauf P, Hervius Askling H, von Sonnenburg F, Lalloo DG, Loutan L, Rapp C, Basto F, Santos O'Connor F, Weld L, Parola P. Infectious diseases among travellers and migrants in Europe, EuroTravNet 2010. Euro Surveill 2012; 17. pii: 20205.

32. Gilden D, White T, Khmeleva N et al. Prevalence and distribution of VZV in temporal arteries of patients with giant cell arteritis. Neurology 2015; 84: 1948-1955.

33. Gnann JW, Sköldenberg B, Hart J et al. National Institute of Allergy and Infectious Diseases Collaborative Antiviral Study Group. Herpes simplex encephalitis: lack of clinical benefit of lon-term valacyclovir therapy. Clin Infect Dis 2015: 61: 683-691.

34. Goenka A, Michael B, Ledger E, Hart I, Absoud M, Chow G, Lilleker J, Lim M, Lunn M, Peake D, Pysden K, Roberts M, Carrol E, Avula S, Solomon T, Kneen R. Neurological manifestations of influenza infection in adults and children: results of a national british surveillance study. J Neurol Neurosurg Psychiatry 2013; 84(11): e2.

35. Goyal M, Demchuk AM, Menon BK et al. Randomized assessment of rapid endovascular treatment of ischemic stroke. N Engl J Med 2015; 372: 1019-1030.

36. Heckenberg SG, Brouwer MC, van der Ende A, van de Beek D. Adjunctive dexamethasone in adults with meningococcal meningitis. Neurology 2012; 79: 1563-1569.

37. Hoffmann B, Tappe D, Höper D et al. A variegated squirrel bornavirus associated with fatal human encephalitis. N Engl J Med 2015; 373: 154-162.

38. Howard JF Jr, Barohn RJ, Cutter GR, Freimer M, Juel VC, Mozaffar T, Mellion ML, Benatar MG, Farrugia ME, Wang JJ, Malhotra SS, Kissel JT. A randomized, double-blind, placebo-controlled phase II study of eculizumab in patients with refractory generalized myasthenia gravis. Muscle Nerve 2013; 48: 76-84.

39. Hufschmidt A, Shabarin V, Zimmer T. Drug-induced confusional states: the usual suspects? Acta Neurol Scand 2009; 120: 436-438.

40. Hughes RA, van Doorn PA. Corticosteroids for Guillain-Barré syndrome. Cochrane Database Syst Rev 2012; 8: CD001446.
41. Jacob S, Viegas S, Leite MI, Webster R, Cossins J, Kennett R, Hilton-Jones D, Morgan BP, Vincent A. Presence and pathogenic relevance of antibodies to clustered acetylcholine receptor in ocular and generalized myasthenia gravis. Arch Neurol 2012; 69: 994-1001.

42. Jovin TG, Chamorro A, Cobo E. Thrombectomy within 8 hours after symptom onset in ischemic stroke. N Engl J Med 2015; 372: 2296-2306.

43. Jüttler E, Unterberg A, Woitzik J, Bösel J, Amiri H, Sakowitz OW, Gondan M, Schiller P, Limprecht R, Luntz S, Schneider H, Pinzer T, Hobohm C, Meixensberger J, Hacke W. Hemicraniectomy in older patients with extensive middle-cerebral-artery stroke. N Engl J Med 2014; 370: 1091-1100.

44. Kawai N, Ikematsu H, Iwaki N, Maeda T, Kanazawa H, Kawashima T, Tanaka O, Yamauchi S, Kawamura K, Nagai T, Horii S, Hirotsu N, Kashiwagi S. A comparison of the effectiveness of zanamivir and oseltamivir for the treatment of influenza A and B. J Infect 2008; 56: 51-57.

45. Kalaria RN, Mukaetova-Ladinska EB. Delirium, dementia and senility. Brain 2012; 135: 2582-2584.

46. Khan F, Pallant JF, Amatya B, Ng L, Gorelik A, Brand C. Outcomes of high- and low-intensity rehabilitation programme for persons in chronic phase after Guillain-Barré syndrome: a randomized controlled trial. J Rehabil Med 2011; 43: 638-646.

47. Kidwell CS, Jahan R, Gornbein J, Alger JR, Nenov V, Ajani Z, Feng L, Meyer BC, Olson S, Schwamm LH, Yoo AJ, Marshall RS, Meyers PM, Yavagal DR, Wintermark M, Guzy J, Starkman S, Saver JL. A trial of imaging selection and endovascular treatment for ischemic stroke. N Engl J Med 2013; 368 : 914-923.

48. Kirkman MA, Mahattanakul W, Gregson BA, Mendelow AD. The effect of the results of the STICH trial on the management of spontaneous supratentorial intracerebral haemorrhage in Newcastle. Br J Neurosurg 2008; 22: 739-746.

49. Koga M, Kusunoki S, Kaida K, Uehara R, Nakamura Y, Kohriyama T, Kanda T. Nationwide survey of patients in Japan with Bickerstaff brainstem encephalitis: epidemiological and clinical characteristics. J Neurol Neurosurg Psychiatry 2012; 83: 1210-1215.

50. Kuitwaard K, de Gelder J, Tio-Gillen AP, Hop WC, van Gelder T, van Toorenenbergen AW, van Doorn PA, Jacobs BC. Pharmacokinetics of intravenous immunoglobulin and outcome in Guillain-Barré syndrome. Ann Neurol 2009; 66: 597-603.

51. Lee SH, Lim GH, Kim JS, Oh SY, Kim JK, Cha JK, Yun CH, Kang JK, Lee H, Song HK, Chung KC. Acute ophthalmoplegia (without ataxia) associated with anti-GQ1b antibody. Neurology 2008; 71: 426-429.

52. Lehmann HC, Hartung HP, Kieseier BC, Hughes RA. Guillain-Barré syndrome after exposure to influenza virus. Lancet Infect Dis 2010; 10: 643-651.

53. Leite MI, Coutinho E, Lana-Peixoto M, Apostolos S, Waters P, Sato D, Melamud L, Marta M, Graham A, Spillane J, Villa AM, Callegaro D, Santos E, da Silva AM, Jarius S, Howard R, Nakashima I, Giovannoni G, Buckley C, Hilton-Jones D, Vincent A, Palace J. Myasthenia gravis and neuromyelitis optica spectrum disorder: a multicenter study of 16 patients. Neurology 2012; 78: 1601-1607.
54. Lizarraga KJ, Alexandre LC, Ramos-Estebanez C, Merenda A. Are steroids a beneficial adjunctive therapy in the immunosuppressed patient with herpes simplex virus encephalitis? Case Rep Neurol 2013; 5: 52-55.

55. Macdonald RL, Kassell NF, Mayer S, Ruefenacht D, Schmiedek P, Weidauer S, Frey A, Roux S, Pasqualin A. Clazosentan to overcome neurological ischemia and infarction occurring after subarachnoid hemorrhage (CONSCIOUS-1): randomized, double-blind, placebo-controlled phase 2 dosefinding trial. Stroke 2008; 39: 3015-3021.

56. Macdonald RL, Higashida RT, Keller E, Mayer SA, Molyneux A, Raabe A, Vajkoczy P, Wanke I, Bach D, Frey A, Marr A, Roux S, Kassell N. Clazosentan, an endothelin receptor antagonist, in patients with aneurysmal subarachnoid haemorrhage undergoing surgical clipping: a randomised, doubleblind, placebo-controlled phase 3 trial (CONSCIOUS-2). Lancet Neurol 2011; 10: 618-625.

57. Macdonald RL, Higashida RT, Keller E, Mayer SA, Molyneux A, Raabe A, Vajkoczy P, Wanke I, Bach D, Frey A, Nowbakht P, Roux S, Kassell N. Randomized trial of clazosentan in patients with aneurysmal subarachnoid hemorrhage undergoing endovascular coiling. Stroke 2012; 43: 1463-1469.

58. Mandawat A, Kaminski HJ, Cutter G, Katirji B, Alshekhlee A. Comparative analysis of therapeutic options used for myasthenia gravis. Ann Neurol 2010; 68: 797-805.

59. Mendelow AD, Gregson BA, Fernandes HM, Murray GD, Teasdale GM, Hope DT, Karimi A, Shaw $\mathrm{MD}$, Barer $\mathrm{DH}$. Early surgery versus initial conservative treatment in patients with spontaneous supratentorial intracerebral haematomas in the International Surgical Trial in Intracerebral Haemorrhage (STICH): a randomised trial. Lancet 2005; 365: 387-397.

60. Mendelow AD, Gregson BA, Rowan EN, Murray GD, Gholkar A, Mitchell PM; STICH II Investigators. Early surgery versus initial conservative treatment in patients with spontaneous supratentorial lobar intracerebral haematomas (STICH II): a randomised trial. Lancet 2013; 382: 397-408

61. Meyding-Lamadé $\mathrm{U}$ et al. Virale Meningitis. In: Diener HC, Weimar C (Hrsg.) Leitlinien für Diagnostik und Therapie in der Neurologie. Deutsche Gesellschaft für Neurologie. Stuttgart: Thieme 2012.

62. Misra UK, Kalita J, Maurya PK. Levetiracetam versus lorazepam in status epilepticus: a randomized, open labeled pilot study. J Neurol 2012; 259: 645-648.

63. Moultrie F, Horne MA, Josephson CB, Hall JM, Counsell CE, Bhattacharya JJ, Papanastassiou V, Sellar RJ, Warlow CP, Murray GD, Al-Shahi Salman R. Outcome after surgical or conservative management of cerebral cavernous malformations. Neurology 2014; 83: 582-589.

64. Molyneux E, Nizami SQ, Saha S, Huu KT, Azam M, Bhutta ZA, Zaki R, Weber MW, Qazi SA. 5 versus 10 days of treatment with ceftriaxone for bacterial meningitis in children: a double-blind randomised equivalence study. Lancet 2011; 377: 1837-1845.

65. Munster BC, Aronica E, Zwinderman AH, Eikelenboom P,Cunningham C, Rooij SE. Neuroinflammation in delirium: a postmortem case-control study. Rejuvenation Res 2011; 14: 615-622. 
66. Naidech AM, Shaibani A, Garg RK, Duran IM, Liebling SM, Bassin SL, Bendok BR, Bernstein RA, Batjer HH, Alberts MJ. Prospective, randomized trial of higher goal hemoglobin after subarachnoid hemorrhage. Neurocrit Care 2010; 13: 313-320.

67. Nguyen TH, Tran TH, Thwaites G, Ly VC, Dinh XS, Ho Dang TN, Dang QT, Nguyen DP, Nguyen HP, To SD, Nguyen VC, Nguyen MD, Campbell J, Schultsz C, Parry C, Torok ME, White N, Nguyen TC, Tran TH, Stepniewska K, Farrar JJ. Dexamethasone in Vietnamese adolescents and adults with bacterial meningitis. N Engl J Med 2007; 357: 2431-340.

68. Oehler E, Watrin L, Larre P, Leparc-Goffart I, Lastere S, Valour F, Baudouin L, Mallet H, Musso D, Ghawche F. Zika virus infection complicated by Guillain-Barre syndrome - case report, French Polynesia, December 2013. Euro Surveill 2014; 19. pii: 20720

69. Peitz GJ, Balas MC, Olsen KM, Pun BT, Ely EW. Top 10 myths regarding sedation and delirium in the ICU. Crit Care Med 2013; 41(9 Suppl 1): S46-56.

70. Petersen LR, Brault AC, Nasci RS. West Nile virus: review of the literature. JAMA 2013; 310: 308-315.

71. Pfefferkorn T, Mayer TE, Opherk C, Peters N, Straube A, Pfister HW, Holtmannspötter M,
Müller-Schunk S, Wiesmann M, Dichgans M. Staged escalation therapy in acute basilar artery occlusion: intravenous thrombolysis and on-demand consecutive endovascular mechanical thrombectomy: preliminary experience in $16 \mathrm{pa}-$ tients. Stroke 2008; 39: 1496-1500.

72. Pfister HW et al. Ambulant erworbene bakterielle (eitrige) Meningoenzephalitis. In: Diener HC, Weimar C (Hrsg.) Leitlinien für Diagnostik und Therapie in der Neurologie. Deutsche Gesellschaft für Neurologie. Stuttgart: Thieme 2012.

73. Popovic N, Miloševic B, Uroševic A, Poluga J, Lavadinovic L, Nedelkovic J, Jevtovic D, Dulovic O Outbreak of West Nile virus infection among humans in Serbia, August to October 2012. Euro Surveill 2013; 18(43): pii=20613.

74. Power KN, Flaatten H, Gilhus NE, Engelsen BA. Propofol treatment in adult refractory status epilepticus. Mortality risk and outcome. Epilepsy Res 2011; 94: 53-60.

75. Ravanini P, Huhtamo E, Ilaria V, Crobu M, Nicosia A, Servino L, Rivasi F, Allegrini S, Miglio U, Magri A, Minisini R, Vapalahti O, Boldorini R. Japanese encephalitis virus RNA detected in Culex pipiens mosquitoes in Italy. Euro Surveill 2012; 17(28): pii: 20221.
76. Rizzo C, Salcuni P, Nicoletti L, Ciufolini MG, Russo F, Masala R, Frongia O, Finarelli AC, Gramegna M, Gallo L, Pompa MG, Rezza G, Salmaso S, Declich S. Epidemiological surveillance of West Nile neuroinvasive diseases in Italy, 2008 to 2011. Euro Surveill 2012; 17(20): pii: 20172.

77. Rossetti AO, Milligan TA, Vulliémoz S, Michaelides C, Bertschi M, Lee JW. A randomized trial for the treatment of refractory status epilepticus. Neurocrit Care 2011; 14: 4-10.

78. Saito K, Shimizu F, Koga M, Sano Y, Tasaki A, Abe M, Haruki H, Maeda T, Suzuki S, Kusunoki S, Mizusawa $\mathrm{H}$, Kanda T. Blood-brain barrier destruction determines Fisher/Bickerstaff clinical phenotypes: an in vitro study. J Neurol Neurosurg Psychiatry 2013; 84: 756-765.

79. Sakurai T, Kimura A, Tanaka Y, Hozumi I, Ogura $S$, Inuzuka T. [Case of adult influenza type A virusassociated encephalopathy successfully treated with primary multidisciplinary treatments] Rinsho Shinkeigaku 2007; 47: 639-643.

80. Santamarina E, Toledo M, Sueiras M, Raspall M, Ailouti N, Lainez E, Porta I, de Gracia R, Quintana M, Alvarez-Sabín J, Puig XS. Usefulness of intravenous lacosamide in status epilepticus. J Neurol 2013; 260: 3122-3128. 